\title{
Perspectives on layout and topology design
}

\author{
J.E. Taylor
}

\begin{abstract}
This note investigates the role of problem formulation in avoiding conputal difficulties. The proposed notion is applied to trusses with stress constraints and to an alternative general approach to topology design.
\end{abstract}

Key words optimal structures, topology design, variational formulation

1

\section{Introduction}

The recent special issue of Structural and Multidisciplinary Optimization (Vol. 21, No. 2) on matters relating to topology and layout design provides a broad summary on modelling and computational experiences in the subject. The idea of the present note is to supplement the special issue with a discussion of material not entirely covered there. As is acknowledged in descriptions furnished in this issue (Rozvany 2001a,b), certain anomalies encountered in practice are not entirely resolved. Present considerations are related to the role of problem formulation in the overall picture, rather than to issues that may arise in connection with computational method. Specifically, the notion is considered that some of the difficulties encountered in the area of computational prediction of design may be avoided with alternate problem formulation. Layout of trusses within stress constraints, and an alternative general approach to topology design are reviewed as two types of design situation where the notion may apply.

Received September 3, 2001

Revised manuscript received January 8, 2002

\section{J.E. Taylor}

Department of Aerospace Engineering, The University of Michigan, Ann Arbor, MI 48109-2118, USA

e-mail: janos@umich.edu

\section{2}

\section{Truss design within stress constraints}

In this example consideration is given to how the portrayal of the associated mechanics may be a critical factor in treatments for structural optimization where limits on stress are to be met. Since with the imposition of stress constraints the kinematical requirement (compatibility condition) of linear elasticity no longer holds, the common displacement-based model for the mechanics is ill suited to the problem. At the same time, limitations on stress state are handled routinely in the setting of a complementary energy representation for elastostatics. Stress appears there as a primary variable, and with stress constraints the complementary energy principle becomes simply a constrained min problem. In this setting, the set of admissible stresses is complete. Also, the multiplier associated with a simple stress constraint, for example, may be identified as the "internal variable" that arises in such problems (a measure of plastic strain in the case of deformation plasticity). Analysis and computational treatment for optimal design are unambiguous using this rendering of the mechanics in the design problem formulation (Taylor 1993b). [The apparent anomaly noted in Example 3, p. 112 of Rozvany $(2001 \mathrm{a}, \mathrm{b})$ does not arise with the stress-based formulation, for example. The complementary energy formulation also might be applicable as an alternative to the approaches described by Cheng and Jiang (1992) and by Kirsch (1990).]

The cited complementary energy formulation, its implementation for computation, and diagrams of example results are available in the Sesimbra paper (Taylor 1993b). In respect of space limitations, the formulation is not repeated here. We note, however, that a load parameter is incorporated in that problem statement. The formulation including load parameter is convenient for treatment of the dependence of design on load state, and as well for the portrayal of problems in design for more general load path. It is applied to advantage in the case of design with nonlinear material, where the optimal 
configuration may include any number of members at or below the stress limit, and in any combination, from among the members provided in the ground structure. Such results are observed in the various examples of the cited paper, including a demonstration of how the optimal layout changes with increasing load. To consider a different aspect of topology design, we note the sort of difficulties that sometimes arise in design settings having symmetries in loading and/or structure (Svanberg and Stolpe 2001) presents examples of this kind]. Here too the facility to interpret dependence of design with respect to continuously varying load parameter may be useful.

The selfsame formulation is applicable in continuum design. There the stress constraint is expressed as a function of the stress tensor (as in statement of a yield condition). An extremum-problem formulation for the analysis of the stress-constrained continuum is given by Taylor (1993a) and applications showing the evolution of response with increasing load are described by Plaxton and Taylor (1994). The formulation extended to cover design has been applied to predict optimal material properties for continuum structures within stress constraints, where the properties vary continuously over the structural domain (Bendsøe et al. 1996). It is applicable as well for the determination of topology design, as suggested in the discussion below.

3

\section{Topology design}

To consider an aspect of problem formulation in the field of topology design of continuum structures, recall that the usual zero-one characterization for that problem (e.g. Ch. 2 Bendsøe 1995) describes an ill-posed problem. The familiar devices such as modification in the analysis by relaxation, or the use of penalization or simulations such as SIMP for computational purposes are required because of this inherent ill-posedness. With these models the problem of 'lack of closure' for the zero-one topology design formulation is circumvented, in effect, by the narrowing of the space of admissible designs. In the case of isotropic plate design, for comparison, a narrowing of the design space via "slope control" (Niordson 1983), i.e. a constraint on the magnitude of the first derivative of the design variable, results in a tractable formulation for that problem. (Notably, Niordson found the slope control model to be relatively inefficient for computational treatment, as did Petersson and Sigmund (1998) for other design problems.) The familiar alternative (for plate design still) has the problem recast into a formulation to accommodate orthotropy (see e.g. Bendsøe 1995), to render it into well-posed form. The properly posed formulation obtained by this broadening of the space of admissible mechanics and designs comprises an effective basis for the overall treatment of solid plate design. To consider for the continuum design a comparable alternative to the narrowing of the design space, the problem formulated in a form where the unrestricted modulus tensor is the design variable (Bendsøe et al. 1994) has closure. This well-posed statement of the continuum problem is fundamental, in the sense that the formulation with pointwise arbitrary material tensor comprises comprehensive coverage for continuum design, within the elasticity model. Stated in another way, design within the mechanics of linear elastostatics is covered for all possible continuum designs by the "unrestricted modulus" formulation. At the same time, computational solutions to problems of this type are obtained using familiar and well-exercised (optimality condition based) methods. Also, design for restricted material properties may be predicted using a suitably constrained version of the unrestricted modulus formulation.

These properties would have the unrestricted modulus interpretation for continuum design be attractive as a basis to predict topology design, and a method to do so (Guedes and Taylor 1997a,b) has proved to be effective in a variety of settings (Rodrigues et al. 1999; Taylor 2000; Du and Taylor 2001, for example). The procedure, termed weighted unit cost method, calls for stepwise evaluation (update) of the optimal material tensor prediction where the unit cost field is modified at each step. To elaborate, the following separate considerations enter into the subject method for topology design.

(a) The method depends on having a proper formulation for design optimization in the setting where distribution of effective material is unrestricted. For truss design, the set of admissible designs would be the ground structure, where member sizes (and possibly member properties) are unrestricted. In the case of continuum structures, this refers to the cited model for design with unrestricted modulus tensor.

(b) Topology or layout design is obtained by a stepwise procedure where at each step unit cost coefficients are adjusted systematically and the model of (a) is applied to predict a new design. This procedure leads to a zero-one or black-white design.

The proper formulation indicated in (a) is simply a properly posed form of problem statement for the analysis and design. In the setting of linear elasticity, this has the convenient form of a min problem with quadratic objective and linear constraints, or its equivalent.

The following features are noted for this procedure to predict topology design.

- The method calls for the solution at each step of the same well-posed design problem, i.e. each stepwise result is an optimal design for the current unit cost field. No relaxation, penalization, ad hoc modification of the model, or other synthetic means are involved. 
- The technique is applicable in the setting of any properly posed design problem and its form is the same in $2 \mathrm{D}$ and $3 \mathrm{D}$.

- Where desired, design results determine the optimal local material properties as well as their distribution. Substantial improvement in performance is realized where the material is optimized along with its topology.

- The method accommodates a variety of practical design goals, e.g. prediction of design where designated areas are to remain void, and determination of designs for specified material constitution (e.g. ortho-tropic, transversely isotropic, isotropic, composites, ...).

- The procedure is computationally stable and relatively efficient. Where checkerboarding might occur, the behavior is controlled by routine method (filtering).

- The model readily admits extension to more general problem settings, e.g. multipurpose design, design for various objectives and constraints, and so on.

- Topology designs are generated using a 97 line program in Matlab (just kidding).

4

\section{Example applications}

Example computational results presented here were obtained as applications of a general purpose program for continuum design. This implementation may be used to predict the optimal spatial distribution and local constitution of material, and the associated topology design. The program used to produce topology solutions comprises an automated form of application of the weighted unit cost method. This particular implementation has

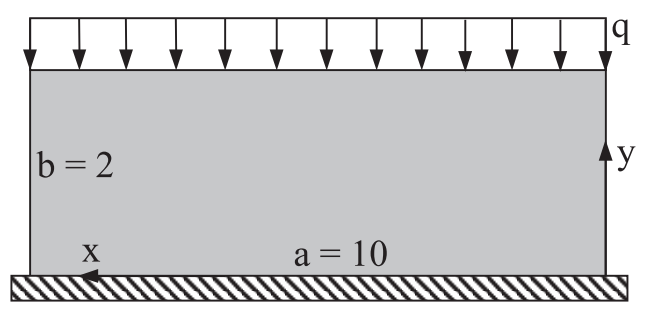

Fixed lower boundary

Fig. 1 Rectangular design domain with lower boundary fully supported

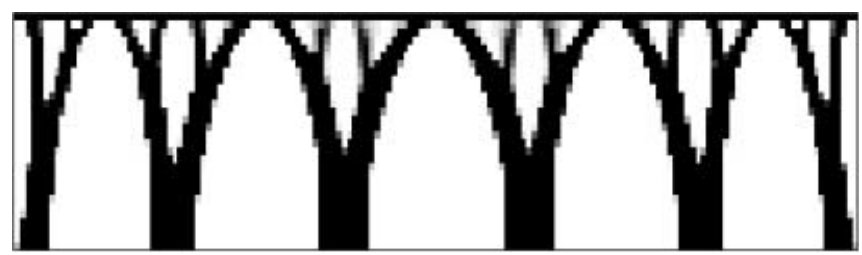

Fig. 2 Multiple-bay results for topology, for the setting of Fig. 1 (all for $33 \%$ volume fraction, isotropic materials)

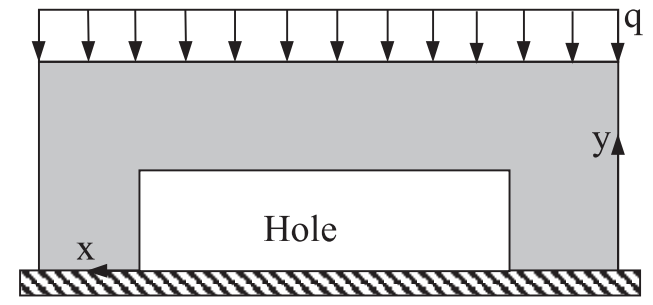

Fixed lower boundary $\quad \mathrm{a}=10$

Fig. 3 Setting as above but with a specified hole as shown

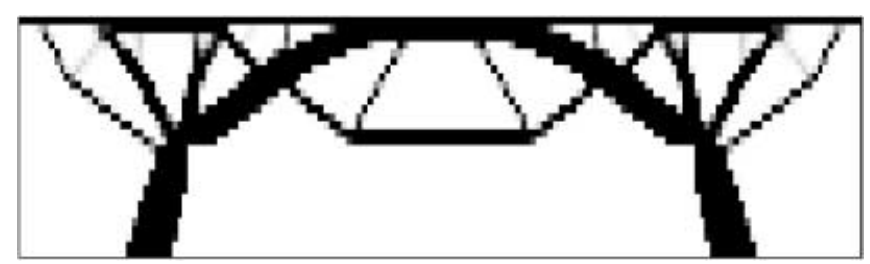

Fig. 4 Topology results for the system of Fig. 3 (33\% volume fraction, isotropic materials)

been created by Dr. Jianbin Du during his tenure as Postdoctoral research fellow at the University of Michigan.

For the example results shown in Figs. 1 to 8 (the structure may be interpreted as a simulation of bridge design), topology diagrams have been produced for a variety of loading and support conditions. The design is for minimum compliance. The provision to predict optimal material properties has been suppressed in these examples, and the material is limited to be isotropic. Availability of continuous support over the span-length leads to the multiple span designs of Fig. 1. The scale for the separate arches in the diagram for this design derives from the computational result, i.e. this feature does not depend on user input of a "local scale". For contrast to the con-

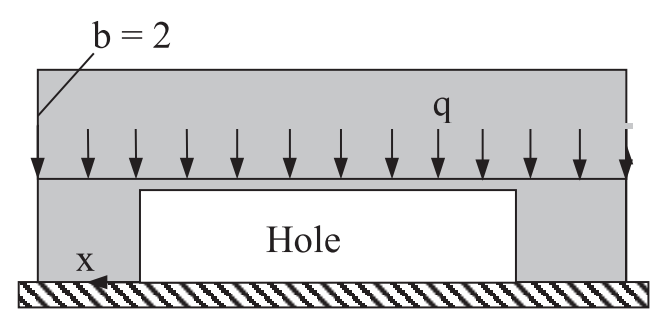

Fixed lower boundary $\quad a=10$

Fig. 5 As above but with Loads moved to mid-height

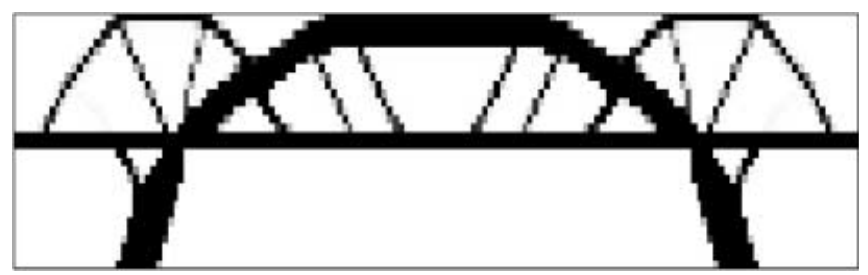

Fig. 6 Topology results for the setting of Fig. 5 (33\% volume fraction, isotropic materials) 


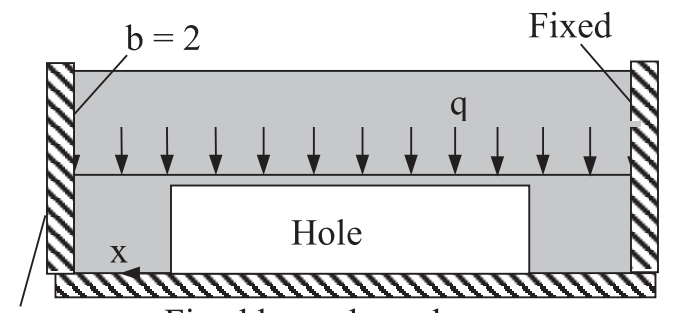

Fixed Fixed lower boundary $\quad \mathrm{a}=10$

Fig. 7 As in Fig. 5 but with both lower and side boundaries supported

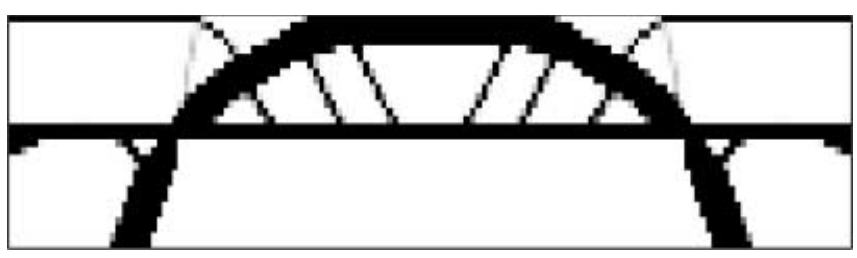

Fig. 8 Topology results for the setting of Fig. 7 (33\% volume fraction, isotropic materials)

tinuous support case, the imposition of requirements for a clear-span leads to a form of super- or substructure. The design results shown in Figs. 4 to 8 indicate subtle changes in design induced by varying the vertical position of the loads and/or by modification in the supports. Additional examples in 2D and 3D and the algorithm implemented into the program that was used to produce them are described by Du and Taylor (2001).

\section{5}

\section{Summary}

Not surprisingly, many alternatives in formulation are possible in the broad approach to the design of continuum structures (e.g. Pedersen 1993; Olhoff et al. 1993, 1997; Foldager 1999, among recent contributions). New and more versatile variational formulations in mechanics are likely to play an important part in future developments for structural and multidisciplinary optimal design. Also, it appears that the weighted unit cost method provides an effective and generally applicable tool for the determination of topology designs, i.e. where the structural resource or material is required to be concentrated, as in "black-white" forms of result.

\section{References}

Bendsøe, M.P. 1995: Optimization of structural topology, shape, and material. Berlin, Heidelberg, New York: Springer

Bendsøe, M.P.; Guedes, J.M.; Haber, R.B.; Pedersen, P.; Taylor, J.E. 1994: An analytical model to predict optimal material properties in the context of optimal structural design. J. Appl. Mech. 61, 930-937
Bendsøe, M.P.; Guedes, J.M.; Plaxton, S.; Taylor, J.E. 1996: Optimization of structure and material properties for solids composed of softening material. Int. J. Solids Struct. 33, 1799-1813

Cheng, G.D.; Jiang, Z. 1992: Study on topology optimization with stress constraint. Eng. Opt. 20, 129-148

Du, J.; Taylor, J.E. 2001: Application of an energy based model for the design of structural materials and topology. Struct. Multidisc. Optim. (to appear)

Foldager, J.P. 1999: Design of composite structures. Ph.D. Dissertation, Special Report No. 39, Institute of Mechanical Engineering, Aalborg University

Guedes, J.M.; Taylor, J.E. 1997a: On the prediction of material properties and topology for optimal continuum structures. Struct. Optim. 14, 193-199

Guedes, J.M.; Taylor, J.E. 1997b: An alternative approach for the prediction of optimal structural topology. Abstracts of ASME, ASCE, SES Summer Meeting (held at Nothwestern University, Evanston, IL)

Kirsch, U. 1990: On singular topology in optimal structural design. Struct. Optim. 2, 133-142

Niordson, F.I. 1983: Optimal design of plates with a constraint on the slope of the thickness function. Int. J. Solids Struct 19, 141-151

Olhoff, N.; Thomsen, J.; Rasmussen, J. 1993: Topology optimization of bi-material structures. In: Pedersen, P. (ed.) Optimal design with advanced materials, pp. 191-206. Amsterdam: Elsevier

Olhoff, N.; Jacobsen, J.B.; Ronholt, E. 1997: Three-dimensional structural topology and layout optimization based on optimum microstructures. Extended Abstracts of WCSMO-2 (held in Zakopane, Poland)

Pedersen, P. 1993: Optimal orientation of anisotropic materials/optimal distribution of anisotropic materials, optimal shape design with anisotropic materials. In: Rozvany, G.I.N. (ed.) Optimization of large structural systems (Proc. NATO/DFG ASI, held in Berchtesgaden, Germany, 1991). Dordrecht: Kluwer

Petersson, J.; Sigmund, O. 1998: Slope constrained topology optimization. Int. J. Numer. Meth. 41, 1417-1434

Plaxton, S.K.; Taylor, J.E. 1994: Application of a generalized complementary energy principle for the equilibrium analysis of softening material. Comp. Meth. Appl. Mech. 117, 91-103

Rodrigues, H.; Soto, C.A.; Taylor, J.E. 1999: A design model to predict optimal two-material composite structures. Struct. Optim. 17, 186-198

Rozvany, G.I.N. 2001a: Aims, scope, methods, history and unified terminology of computer-aided topology optimization in structural mechanics. Struct. Multidisc. Optim. 21, 90-108

Rozvany, G.I.N. 2001b: Stress ratio and compliance based methods in topology optimization - a critical review. Struct. Multidisc. Optim. 21, 109-119 
Svanberg, K.; Stolpe, M. 2001: On the formulation of topology optimization problems. In: Bendsøe, M.P.; Olhoff, N.; Rasmussen, J. (eds.) Proc. 2-nd Max Planck Workshop on Engineering Design Optimization (held in Nyborg, Denmark)

Taylor, J.E. 1993a: A global extremum principle for the analysis of solids composed of softening materials. Int. J. Solids Struct. 30, 2057-2069
Taylor, J.E. 1993b: Truss topology design for elastic/softening materials. In: Bendsøe, M.P.; Mota Soares, C.A. (eds.) Topology design of structures. Dordrecht: Kluwer

Taylor, J.E. 2000: A formulation for optimal structural design with optimal materials. In: Rozvany, G.I.N.; Olhoff, N. (eds.) Topology optimization of structures and composite continua, pp. 49-59. Dordrecht: Kluwer 